\title{
High Pressure Freezing (HPF) using the Compact HPF 01 unit and Evaluation of Freeze-Substitution Methods for Cyanobacteria and Plant Cells
}

\author{
D.M. Sherman* and C.P Huang*
}

*Purdue University, Life Science Microscopy Facility, 170 S. University, West Lafayette, IN 47906

The advantages of immobilizing and cryo freezing cells under high pressure is well documented for both animal and plant material [1]. While the majority of published results using high pressure freezing were done using the Baltec HPM 010 high-pressure freezer, numerous examples are now appearing in the literature using the Leica EM HPF. A third unit, the Compact 01 HPF unit (Figure 1), manufactured by M. Wohlwend $\mathrm{GmbH}[2]$ and distributed through Technotrade Inc., (Manchester, NH), is now available and was utilized for this research.

The mechanism of the new Compact HPF 01 is similar to that of the current Baltec HPM 010 using similar planchettes and capable of freezing samples up to $0.2 \mathrm{~mm}$ thick and $0.6 \mathrm{~mm}^{3}$ volume. However, the unit has a much smaller footprint and more competitive pricing. In addition, it is much more nitrogen efficient with 50L of liquid nitrogen sufficient for initial cooling and approximately 40 sample "shots" over a period of $2+$ hours. It is not as portable as the Leica EM HPF, but the ease of use, increased sample size, footprint size, nitrogen efficiency, mechanism of action, and pricing all combine to make it a competitive unit for cryo sample preparation.

We have tested the unit using cyanobacteria, Arabidopsis leaves, and plant suspension cells. Plant cells were chosen for the initial testing due to the unique challenges of the dense cell walls, and large internal vacuoles and air spaces for both freezing and later freeze-substitution and resin infiltration. Results were highly encouraging with most initial samples being well frozen with little visible ice damage.

However, the best HPF sample is also only as good as the freeze substitution (FS) that follows. This has proven to be the limiting factor in our sample preparation. We chose the cyanobacterium Cyanothece Sp ATCC 51142 as an initial test organism as we have documented its structure using plunge-freezing [3]. Well over $90 \%$ of the cells frozen using the Compact 01 demonstrated excellent ultrastructure with little sign of freezing damage. This is a substantial improvement over our plunge freezing results when, in particular, we were unable to preserve dividing cells without some ice damage. This particular cyanobacterial strain contains extensive photosynthetic lamellae but also is capable of fixing atmospheric nitrogen and storing this fixed nitrogen as the amino acid polymer cyanophycin until needed. It also has a strong circadian rhythm and glycogen, stored in the form of granules, is produced during the light period for later utilization as an energy source during the dark period. We found that the cell morphology was excellent when $\mathrm{FS}$ in acetone $+2 \% \mathrm{OsO}_{4}$. However, the photosynthetic membranes were only clearly visible when the acetone $/ \mathrm{OsO}_{4}$ was removed at $-20^{\circ} \mathrm{C}$ prior to the final sample warm-up (Figure 2). Under these conditions, the glycogen granules showed minimal substructure. If the cells were left in the substitution media until warmed to $25^{\circ} \mathrm{C}$, the granules had substantial internal staining and structure, whereas the membranes appeared in negative contrast (Figures 3,4).

We intend to use these cells for electron tomography to gain further insight into the interaction of the photosynthetic lamellae with the granules. It is thus critical that both membranes and granules be visible with the best possible preservation. Modifications to the warm-up procedure, including additions of uranyl acetate and tannic acid, will be presented for discussion [4]. 


\section{References:}

[1] S. Craig and LAStaehelin, Eur J Cell Biol (1988) 46: 80-93

[2] We would like to thank Technotrade, International (Manchester, $\mathrm{NH}$ ) for providing access to the Compact HPF 01.

[3] KJ Reddy et al, J. Bacteriol. (1993) 175:1284-1292.

[4] We would like to acknowledge Louis Sherman and Nicholas Carpita (both from Purdue University) for providing sample material and Jothan Austin (Univ. of Chicago) for suggesting alternative FS protocols.
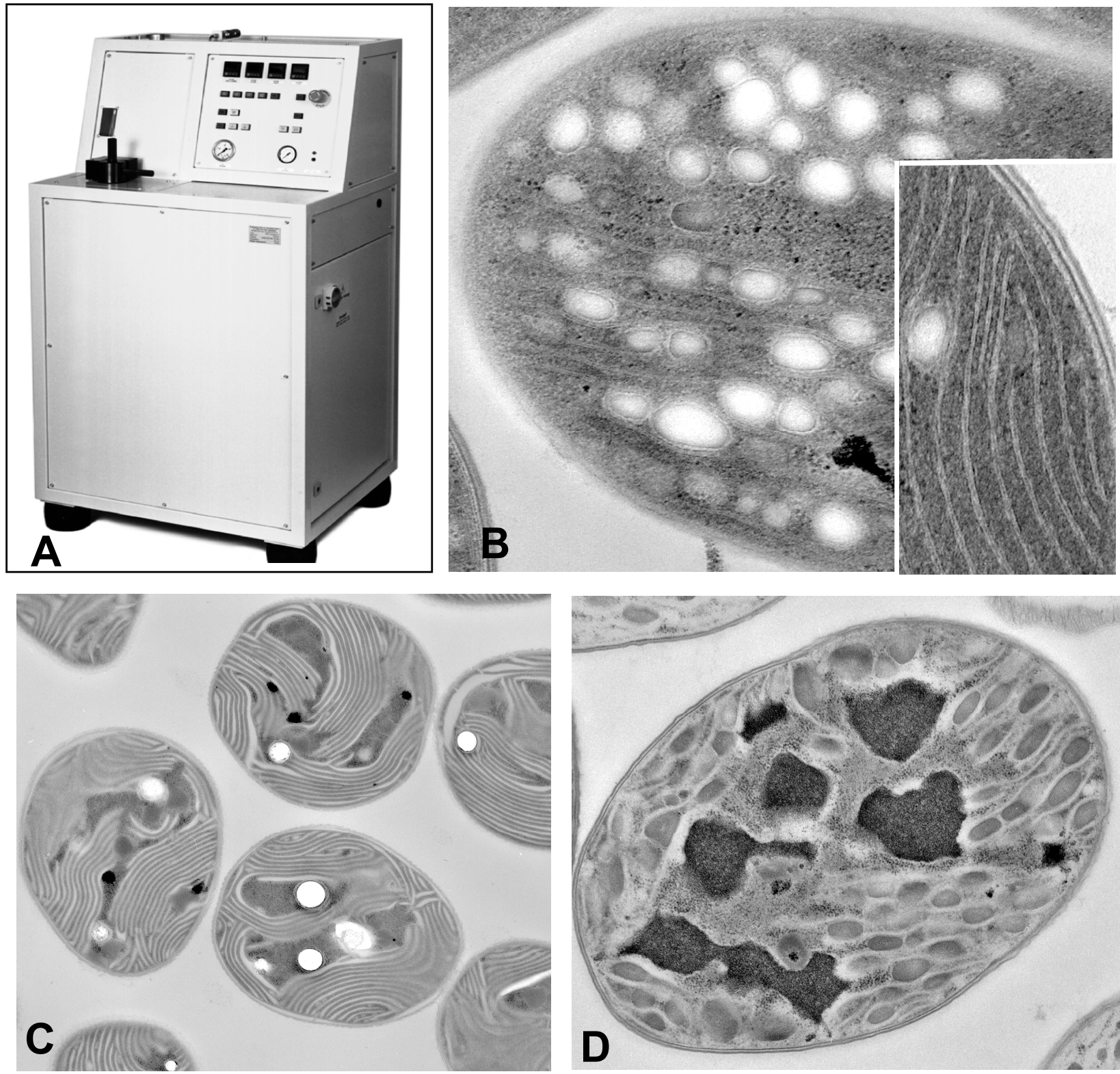

A. The Wohlwend Compact HPF 01

B. Cyanobacteria has photosynthetic membranes (inset) but lacks staining in glycogen granules.

C. Cells grown with nitrogen and illustrating negative contrast in membranes.

D. Cells grown under nitrogen-fixing conditions show excellent preservation of glycogen granules and cyanophycin reserves but lack well stained membranes. 